\section{A TRAJETÓRIA DE VIDA DO TREINADOR ESPORTIVO: AS SITUAÇÕES DE APRENDIZAGEM EM CONTEXTO INFORMAL}

\author{
LIFE TRAJECTORIES OF SPORTS COACHES: LEARNING SITUATIONS IN \\ INFORMAL CONTEXTS
}

\author{
LA TRAYECTORIA DE VIDA DEL ENTRENADOR DEPORTIVO: LAS \\ SITUACIONES DE APRENDIZAJE EN CONTEXTO INFORMAL
}

\author{
Vinicius Zeilmann Brasil", Valmor Ramos **, Thais Emanuelli da Silva de Barros*, \\ Jonas Godtsfriedt*, Juarez Vieira do Nascimento*
}

\begin{abstract}
Resumo: O presente ensaio procurou caracterizar e discutir os contextos e situações de aprendizagem de treinadores esportivos, estabelecendo uma trajetória a partir de episódios significativos neste processo. A literatura específica indica que os treinadores têm valorizado diversas fontes de conhecimento, destacadamente as experiências de prática como atleta, a observação de seus pares, o compartilhamento de informações e a reflexão sobre sua prática. A aprendizagem profissional do treinador pode ser entendida como um processo de socialização esportiva que inicia na infância e se prolonga ao longo da vida, em que prepondera a participação do treinador em situações de aprendizagem em contexto informal.
\end{abstract}

Abstract: This essay sought to characterize and discuss sport coaches' learning contexts and situations, establishing a trajectory after significant episodes in that process. Specific literature indicates that coaches have emphasized different sources of knowledge, notably practice experiences as athletes, observing their peers, sharing information, and reflecting on their practice. Coaches' professional learning can be understood as a process of sports socialization that begins in childhood and continues throughout life, where their participation focuses on learning situations in informal contexts.

Resumen: Este ensayo intenta caracterizar y discutir los contextos y situaciones de aprendizaje de entrenadores deportivos, estableciendo una trayectoria a partir de episodios significativos en este proceso. La literatura específica indica que los entrenadores han valorado diversas fuentes de conocimiento, en particular las experiencias prácticas como atleta, la observación de sus pares, el intercambio de información y la reflexión sobre su práctica. El aprendizaje profesional del entrenador puede ser entendido como un proceso de socialización deportiva que empieza en la infancia y sigue durante toda la vida, en el que predomina la participación del entrenador en situaciones de aprendizaje en un contexto informal.
*Universidade Federal de Santa Catarina (UFSC). Florianópolis, SC. Brasil.

E-mail: vzbrasil@hotmail.com

**Universidade do Estado de Santa Catarina (UDESC). Florianópolis, SC. Brasil.

E-mail: valmor.ramos@udesc.br

Recebido em: 10-10-2014

Aprovado em: 05-06-2015

(c) (i) () Licence 


\section{INTRODUÇÃOO}

A busca pela qualidade na formação profissional e na intervenção pedagógica no âmbito do esporte tem incentivado a realização de investigações empíricas a fim de identificar as particularidades do percurso de aprendizagem de treinadores esportivos experts (CUSHION; NELSON, 2013). Entre as diversas expressões (coach learning, coach education, coach training) utilizadas na literatura internacional para designar o processo de desenvolvimento profissional do treinador (coach development), particularmente, a expressão "coaching learning" faz referência a uma linha de investigação sobre "como 0 treinador aprende a ser treinador" (NELSON; CUSHION; POTRAC, 2006).

As pesquisas sobre essa temática buscam descrever e interpretar o modo como os treinadores constroem seus conhecimentos para intervir no esporte, tomando como referência sua própria trajetória de vida (JONES; ARMOUR; POTRAC, 2003). Estes estudos têm sido realizados com treinadores experts de diferentes modalidades, a exemplo, 0 basquetebol (RAMOS et al., 2011), hóquei no gelo (WRIGHT; TRUDEL; CULVER, 2007), ginástica esportiva (IRWIN; HANTON; KERWIN, 2004), futebol (TALAMONI; OLIVEIRA; HUNGER, 2013), surfe (RAMOS; BRASIL; GODA, 2012); e o handebol (CUNHA; ESTRIGA; BATISTA, 2014).

Ao serem acessadas por meio da combinação de procedimentos qualitativos de pesquisa, as informações proporcionam uma descrição detalhada da trajetória pessoal e profissional dos treinadores. Os principais procedimentos utilizados são as entrevistas, observações sistemáticas, notas de campo e pensamento em voz alta, que consiste em uma entrevista que visa estimular e registrar a verbalização ou relato de uma sequência de pensamentos acerca de experiências, situações, episódios ou ações realizadas (CUSHION et al., 2010).

O reconhecimento dos episódios da vida e da identidade desses indivíduos pode originar reflexões importantes para formação profissional, sobretudo no que diz respeito ao processo de aprender a ensinar e de tornar-se treinador (CALLARY; WERTHNER; TRUDEL, 2012).

De acordo com a proposta das metáforas de aprendizagem de Sfard (1998), os treinadores aprendem a ensinar a partir de dois possíveis processos: através da metáfora da aquisição e/ou da metáfora da participação. Enquanto que a aprendizagem na metáfora da aquisição é caracterizada por situações de transferência direta da mente de um indivíduo conhecedor para outro, do que os treinadores "devem saber" e "devem fazer", a aprendizagem na metáfora da participação resulta de situações onde há um engajamento pessoal do indivíduo para interagir com outras pessoas a partir de seus próprios interesses, caracterizando a aprendizagem como uma atividade de proporções sociais (SFARD, 1998, TRUDEL; GILBERT, 2006).

De fato, o processo de tornar-se treinador está vinculado diretamente à trajetória de vida pessoal do indivíduo, de modo que a reconstrução e a descrição deste percurso pelo próprio treinador podem revelar episódios, decisões e opções circunstanciais, dotadas de significados e informações úteis para se estabelecer direcionamentos e ações para 0 desenvolvimento profissional de futuros treinadores (JONES; ARMOUR; POTRAC, 2004). Embora os resultados dos estudos forneçam informações a respeito de realidades 
particulares, percebe-se que a construção do conhecimento do treinador é "balizada" por um contexto sociocultural de prática esportiva, no qual as interações estabelecidas com os demais membros desse grupo social são fundamentais. Além disso, o engajamento espontâneo dos treinadores para a aprendizagem de um domínio específico de conhecimento está vinculado ao compartilhamento de interesses comuns nessas interações (NELSON; CUSHION; POTRAC, 2006).

Diante do exposto, e tendo como referência a literatura a respeito da aprendizagem do treinador esportivo (coach learning), o objetivo deste ensaio é caracterizar e discutir a respeito dos contextos e situações de aprendizagem mais valorizados pelos treinadores, buscando indicar episódios relevantes na aquisição de conhecimentos para a intervenção profissional.

\section{APONTAMENTOS INICIAIS PARA UMA INTERPRETAÇÃO DA APRENDIZAGEM DO TREINADOR}

Ao considerar a aprendizagem um processo de mudança constante do indivíduo, devese atribuir certa importância aos elementos "tempo" e "espaço", ainda mais pela influência atual da globalização e da tecnologia na quantidade e qualidade das oportunidades de aprendizagem (TRUDEL; CULVER; WERTHNER, 2013).

No âmbito da aprendizagem do treinador, o cenário em que esta ocorre, seja ele um curso, uma instituição, federações, ou qualquer outro ambiente, corresponde aos contextos em que 0 treinador aprende, os quais são representados pelas expressões Formal, Não formal e Informal, propostas por Cushion, Nelson e Potrac (2006).

A percepção particular do aprendiz (treinador) sobre o que ele "vive" nestes contextos é o que tem sido denominado de situações de aprendizagem, como exemplo, a interação com outras pessoas, as experiências de prática, a reflexão, leitura, observação etc. Werthner e Trudel (2006) sugerem três situações gerais de aprendizagem: Mediada, Não mediada (Direta) e Interna, as quais não se referem a diferentes formas de aprendizado mas sim a situações distintas de aprendizagem, a partir da perspectiva do próprio treinador (aprendiz).

Considerando, portanto, o tornar-se treinador um processo de aprendizagem que ocorre ao longo da vida, deve-se ter em conta que o aprendiz carrega consigo um conjunto de conhecimentos obtidos a partir de suas experiências pessoais, da socialização em família, na escola, no trabalho, bem como suas concepções e perspectivas pessoais para seguir aprendendo.

Os parágrafos que seguem irão discorrer particularmente sobre as experiências esportivas do treinador antes de se tornar treinador e, ainda, a respeito das situações de aprendizagem típicas do contexto informal.

\section{AS EXPERIÊNCIAS PESSOAIS DE PRÁTICA ESPORTIVA DO TREINADOR}

A Figura 1 sugere uma representação da trajetória de prática pessoal do treinador esportivo, indicando as situações de contatos iniciais com o esporte até o momento das primeiras experiências no papel de treinador. 
Figura 1 - Trajetória de experiência esportiva do treinador

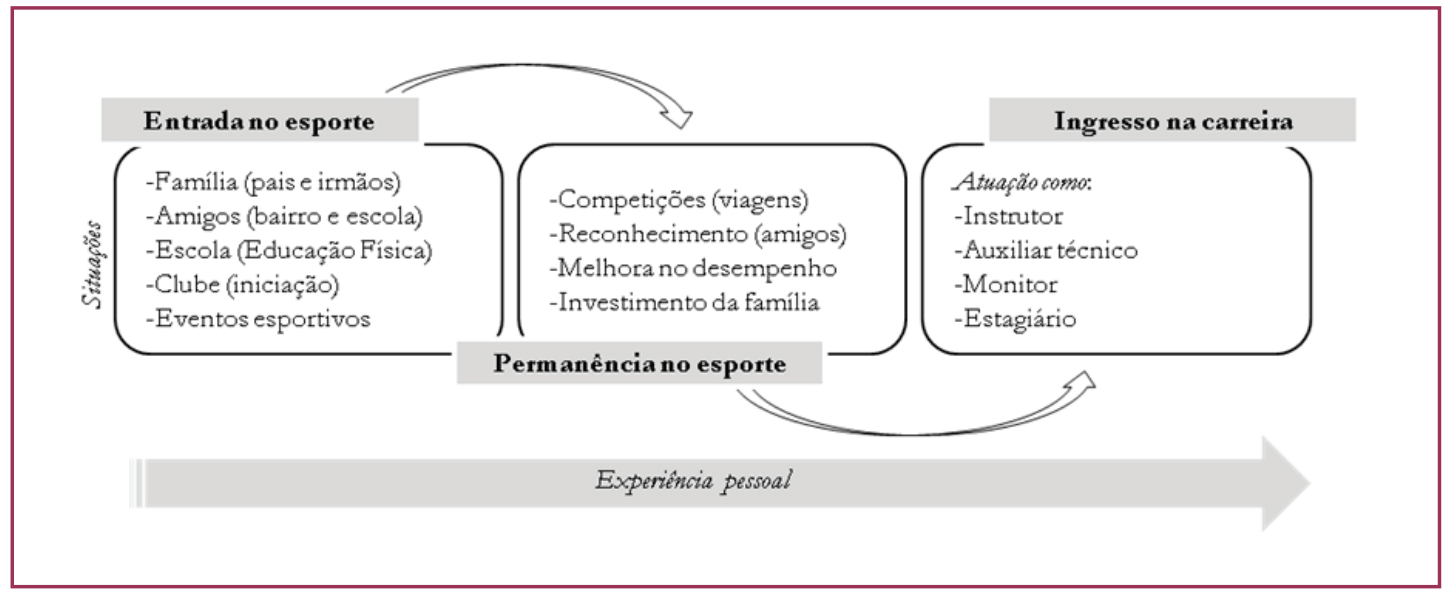

Fonte: Do autor

As primeiras experiências e a inserção dos treinadores no contexto esportivo geralmente estão associadas aos episódios ou situações de convívio sociocultural específicos de prática esportiva, proporcionados principalmente pelos familiares (pais, irmãos, primos, tios, entre outros). Essas experiências que ocorrem ainda na infância têm, predominantemente, um caráter lúdico, de lazer, ligado aos contextos de relações sociais mais imediatos (CÔTÉ; HAY, 2002), proporcionados pelos pais, os quais acabam por fornecer condições e recursos necessários para a manutenção de uma prática esportiva sistematizada (FRASER-THOMAS; CÔTÉ, 2009).

A influência de ex-atletas, através de vínculos familiares ou amizade, pode direcionar a prática de determinado esporte. Nos clubes, associações e outras organizações similares, por meio de aulas extracurriculares, é possivvel obter os primeiros contatos com a prática esportiva sistemática. Além disso, as aulas de Educação Física Escolar e os eventos esportivos muitas vezes podem despertar o interesse pela prática.

Segundo Schwartz (2005), os contextos familiar e de amizades são geralmente os ambientes sociais iniciais e centrais para as crianças, sendo responsáveis pela internalização de significados que servirão de base para a formação de todos os demais pensamentos, sentimentos e comportamentos ao longo da vida. De fato, a socialização esportiva, através desses ambientes, apresenta-se como um processo pelo qual as pessoas adquirem comportamentos e crenças ou a cultura do meio social no qual elas vivem, sendo a família, amigos, comunidade, escola, meios de comunicação, sistema de leis, normas e crenças sociais as principais fontes de socialização (CÔTÉ; HAY, 2002).

No decorrer do envolvimento da criança com a prática esportiva, alguns aspectos reguladores dessa participação são fundamentais para o seu engajamento em fases posteriores (adolescência e juventude). Bailey, Cope e Pearce (2013) comentam que estudos indicam uma variedade de fatores que podem facilitar a participação infantil no esporte, incluindo o incentivo por parte da família, o acesso aos locais de prática, a participação em ambientes que favorecem experiências esportivas positivas (em nível escolar), o reconhecimento dos amigos e a melhoria das habilidades esportivas.

Em particular, a aquisição de novas habilidades, possibilitada pela prática sistemática, pode fazer com que o praticante desenvolva, inicialmente, o que tem de mais hábil, levando-o a ser reconhecido perante o grupo de amigos, familiares e professores como um praticante 
habilidoso. Para Keegan et al. (2009), a valorização pessoal por meio das relações sociais pode envolver e estimular os praticantes a permanecer no esporte por diferentes motivos: pela aprendizagem de novas habilidades; formação de grupos de amizade, quando muitas vezes 0 estar perto dos amigos torna-se mais importante do que a própria prática esportiva; participação em competições, que desafia a capacidade do praticante e o torna diferente ao receber uma premiação ou recompensa pelo seu desempenho; envolvimento dos familiares, que por históricos de esportistas na família o incentivam a continuar praticando; relação com o professor/treinador, que o faz sentir valorizado e reconhecido perante os demais; pelas viagens, sendo para alguns a única oportunidade de conhecer outros lugares, outros atletas, outras culturas; e ainda por uma busca individual de melhora em determinadas habilidades esportivas, desencadeada pela participação em atividades onde haja a interação com outros indivíduos mais habilidosos.

Essas experiências de prática durante a infância e a juventude podem possibilitar ao indivíduo as primeiras representações sobre o que é ser treinador, as quais servirão de referência para as escolhas futuras (SALMELA, 1994), isto é, as ideias, vontades e interesses são modelados a partir de suas experiências, principalmente daquelas que se apresentaram no interior dos grupos em que conviveu ao longo de sua vida.

Quando atinge um estágio avançado de prática, o jovem aproxima-se intensamente do papel de treinador, sobretudo pelo conhecimento que adquire a respeito da prática e pelo seu envolvimento em uma comunidade esportiva específica. A transição de esportista/atleta para treinador se dá concretamente através das primeiras experiências como instrutor, auxiliar técnico, estagiário ou monitor, as quais definem e/ou aprimoram sua capacidade para assumir o papel de treinador (SCHINKE; BLOOM; SALMELA, 1995).

Talamoni, Oliveira e Hunger (2013) destacam que essas experiências correspondem a um momento de reflexão do atleta/treinador referente à escolha de sua profissão, após encerrar a sua carreira como atleta, ou seja, um período favorável à escolha e ao ingresso em um contexto mais formal (cursos de ensino superior ou programas de formação de treinadores) de qualificação para atender as necessidades formativas e normativas exigidas ao exercício legal da profissão de treinador esportivo. 0 treinador aspirante, neste caso, desencadeia um processo de confronto entre o conhecimento específico sobre o esporte adquirido com a prática; suas experiências recentes como auxiliar/estagiário; e ainda, o acesso a novos conhecimentos por meio de livros, clínicas e seminários para treinadores (SCHINKE; BLOOM; SALMELA, 1995).

O ingresso na carreira de treinador esportivo, em parte, está associado à exposição prolongada das pessoas, desde a infância, a um ambiente de prática esportiva, favorecendo a aprendizagem de elementos particulares dessa cultura específica, ou seja, um tipo de "aculturamento" esportivo. Trata-se de um processo "natural" de envolvimento e participação em ambientes e situações variadas, o que confere um caráter sociocultural à aprendizagem (TRUDEL; GILBERT, 2006).

\section{CONTEXTO INFORMAL COMO REFERÊNCIA NA AQUISIÇÃO DO CONHECIMENTO DO TREINADOR}

Segundo Nelson, Cushion e Potrac (2006) a aprendizagem dos treinadores pode ser interpretada a partir de três contextos possíveis, nomeadamente os contextos Formal, 
Não formal e Informal. Cada um tem características próprias, situações típicas e comuns, de aprendizagem (Figura 2).

Figura 2 - Contextos e situações de aprendizagem profissional dos treinadores

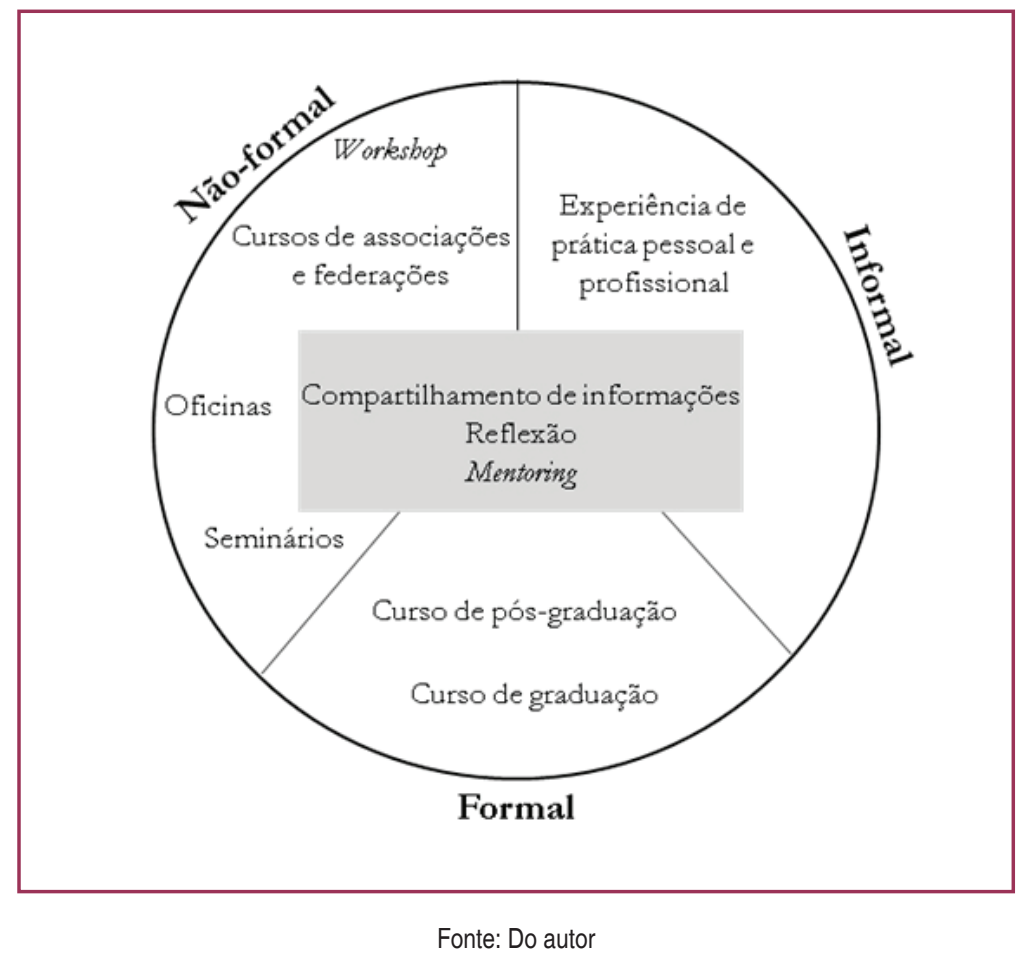

Segundo Cushion et al. (2010), apesar dos treinadores reconhecerem a importância da aprendizagem em contextos formais e não formais, a validade ecológica dos programas e cursos de formação profissional tem sido questionada para favorecer a aquisição de conhecimentos úteis, sendo que as situações de aprendizagem em contextos informais, na percepção dos próprios treinadores, são as que têm maior impacto ou significado no processo de aprendizagem profissional.

O contexto informal corresponde aos ambientes que proporcionam situações de aprendizagem contínua, ao longo da vida, onde cada indivíduo constrói crenças, conhecimentos, atitudes e discernimentos próprios a partir de experiências do cotidiano. 0 envolvimento nesses contextos depende, em parte, de um engajamento pessoal de busca por uma melhor qualificação profissional (NELSON; CUSHION; POTRAC, 2006).

Por conseguinte, há uma valorização das situações ligadas ao cotidiano de prática esportiva, isto é, as experiências esportivas que antecederam o ingresso na carreira de treinador; e de situações ligadas à intervenção do treinador, sobretudo, o mentoring, a experiência como treinador (reflexão) e o compartilhamento de informações (aprendizagem situada) com os pares.

A reflexão indica uma situação de aprendizagem interna resultante de um processo de "olhar para trás" em que não há nenhum novo material de aprendizagem, em que o indivíduo reorganiza o que já sabe, busca o sentido do que aconteceu e identifica novas possibilidades, a fim de melhorar a prática no futuro (breve ou não) (WERTHNER; TRUDEL, 2006). Já 0 mentoring e o compartilhamento de informações representam situações de aprendizagem não mediadas (diretas) em que os aprendizes (treinadores) decidem quais as informações que thes são úteis. Essas situações podem proporcionar, por um lado, um aprendizado inconsciente 
simplesmente pelo fato de 0 aprendiz estar em um ambiente que favorece a aprendizagem de elementos específicos (por exemplo, aprender a subcultura de um esporte); e, por outro, uma aprendizagem consciente através do esforço realizado pelos próprios treinadores para encontrar e selecionar as informações úteis à resolução dos dilemas da prática profissional (WERTHNER; TRUDEL, 2006).

\subsection{Mentoring}

O mentoring pode ser entendido como um processo de interação entre dois indivíduos em que um deles é mais experiente (mentor), servindo de modelo, e orienta a aprendizagem de um principiante, favorecendo a aprendizagem individual para desempenhar determinada profissão (WEAVER; CHELLADURAI, 1999). No caso da aprendizagem do treinador, esse processo pode ser desenvolvido intencionalmente a partir de uma estrutura formal estabelecida a priori, ou então pode ocorrer de modo informal, de acordo com as disposições pessoais dos indivíduos envolvidos no processo.

Neste último caso, Soler (2003) indica que o mentoring informal resulta de um interesse individual e voluntário, em que normalmente existe algum tipo de vínculo entre o mentor e 0 aprendiz, quando há o compartilhamento de experiências, ideias, competências e habilidades, pautadas em uma relação socioafetiva. Dentre os exemplos mais recorrentes desse tipo de "mentoria", Cassidy, Jones e Potrac (2004) e Cushion (2006) destacam as experiências como assistente técnico, auxiliares e também como instrutores, que geralmente ocorrem no período de transição entre a fase de esportista/atleta e o ingresso na carreira de treinador. Assim, 0 processo de mentoring se concretiza na medida em que 0 aprendiz de treinador acompanha e observa o trabalho diário do treinador principal (mais experiente) por algum tempo.

Ao observar o treinador experiente (mentor), o treinador mais jovem é influenciado, aceita e aprende voluntariamente ou involuntariamente modelos de intervenção mais elaborados, principalmente quando ainda está ingressando na carreira profissional (GILBERT; TRUDEL, 2001, CUSHION; ARMOUR; JONES, 2003). Esse modo de aprendizagem tem sido interpretado também a partir do processo de modelação do comportamento, proposto na Teoria Social Cognitiva de Bandura (2008), a qual indica que os comportamentos que uma pessoa domina decorrem da observação que realiza de outras pessoas mais prestigiadas, referenciadas como um modelo positivo.

O sucesso da aprendizagem, neste caso, depende da qualidade da interação estabelecida entre o mentor e o aprendiz, mas a falta de tempo dos indivíduos, a falta de afinidade pessoal ou profissional e as atitudes ou os comportamentos indesejáveis podem ser considerados os problemas mais comuns que afetam negativamente o processo de mentoring (SOLER, 2003).

\subsection{Experiência como treinador (reflexão)}

A aprendizagem dos treinadores através da experiência parece ser "decifrada", em parte, a partir dos mecanismos envolvidos no processo de reflexão (CUSHION; ARMOUR; JONES, 2003), embora não se tenha até o momento uma fundamentação empírica consistente que possa evidenciá-la (CUSHION; NELSON, 2013). 
O conceito de reflexão, no processo de aprendizagem do treinador, é consensualmente abordado com base nas proposições de Schön (2000) a respeito do que denominou de "epistemologia da prática", e da noção de "profissional reflexivo". Na perspectiva da epistemologia da prática o profissional é um elemento ativo, de modo que suas decisões e ações são centrais na elaboração do seu próprio conhecimento profissional. A adoção desse paradigma implica a valorização de um conhecimento prático, obtido a partir da experiência pessoal, anteriormente negligenciado no contexto científico.

Neste sentido, o termo "profissional reflexivo" indica que o conhecimento é obtido a partir de um processo contínuo e sistemático de reflexão, decorrente da resolução de situações problemáticas e reais de prática, traduzindo, assim, a aprendizagem profissional em um processo contínuo de reconstrução das próprias experiências pessoais. No caso da intervenção do treinador esportivo, eles podem aprender engajando-se em três possíveis formas de prática reflexiva: através da reflexão-na-ação, que ocorre durante a ação do treinador; reflexão-sobrea-ação, acontece no momento presente, mas não em meio à intervenção do treinador; e a reflexão-sobre-a-reflexão-na-ação, realizada fora do presente da ação.

No que diz respeito à reflexão-na-ação, o conhecimento é construído a partir de um ciclo de pensamento do treinador ao se deparar com um problema ou uma situação dilemática, estimulando a criação espontânea de uma estratégia para solucioná-la. Esse mecanismo ocorre simultaneamente à intervenção do treinador, podendo interferir na situação quando ele ainda está agindo e, por diversas vezes, alterando e recriando novas formas de fazer até que o êxito de solucionar o problema seja alcançado. Já a reflexão-sobre-a-reflexão-naação é bastante utilizada por meio da análise de vídeos, diários de campo ou anotações de desempenho para que o treinador possa avaliar, repensar e planejar suas metas de trabalho e, consequentemente, suas intervenções futuras.

Esse processo resulta em um tipo de conhecimento prático também denominado de conhecimento tácito ou procedimental, que tem origem na prática profissional, elaborado no confronto com as situações reais de intervenção, o que confere a ele um caráter pessoal, que inclui intenções e propósitos particulares, contextualizado, orientado à solução de problemas e de difícil codificação (MONTERO, 2005, NASH; COLLINS, 2006). Acredita-se, portanto, que a prática reflexiva permite ao treinador aprender por meio de suas próprias experiências enquanto treinador, oferecendo a possibilidade de alterar e adaptar melhor suas ações em resposta aos novos dilemas, com base no que foi aprendido anteriormente (TRUDEL; GILBERT, 2006).

Embora a contemplação, avaliação e ponderação também correspondam a processos de pensamento que têm sido utilizados para indicar a reflexão, tais procedimentos não representam a prática reflexiva com todos seus atributos (CROPLEY; HANTON, 2011). As evidências iniciais indicam que a reflexão superficial pode não ser eficaz como um meio de aprendizagem, ou seja, a mudança ou a reconstrução de crenças, conhecimentos e atitudes dos treinadores devem se dar em um nível de reflexão profunda e sistemática (MOON, 2004).

\subsection{Compartilhamento de informações (aprendizagem situada)}

A partir dos postulados da teoria da aprendizagem situada de Lave e Wenger (1991), a aprendizagem dos treinadores esportivos pode ser entendida como uma prática social complexa e dinâmica em que o "aprender" é uma de suas características fundamentais, estabelecida 
entre os indivíduos no decorrer da vida. Assim, o treinador aprende por meio das interações que estabelece com os indivíduos pertencentes a um mesmo grupo, com interesses em comum e que compartilham práticas, atitudes, valores, crenças e conhecimentos (CULVER; TRUDEL, 2006), dando origem ao que tem sido denominado de Comunidades de Prática (WENGER, 1998).

As Comunidades de Prática correspondem a grupos ou redes de pessoas e/ou profissionais (treinador) ou próximas (dirigentes, atletas, ...), ligados formal ou informalmente para compartilhar um interesse ou a "paixão" por algo, que interagem regularmente para aprender mais a respeito de um domínio especializado comum ao grupo. Os membros de uma comunidade compartilham experiências e conhecimento com liberdade e criatividade, incentivando novas abordagens para a solução dos problemas da prática, além disso, regulam sua própria prática em conformidade com normas de conduta e proficiência, consensuadas pela comunidade (WENGER, 1998).

Para Wenger (1998), uma Comunidade de Prática se estabelece a partir de quatro componentes: do significado, que traduz a necessidade que cada indivíduo tem de encontrar um sentido para o mundo; da prática social, que exprime a experiência compartilhada de recursos e perspectivas que mantêm o envolvimento mútuo dos membros na ação; da comunidade, que corresponde à configuração social na qual se definem as iniciativas e onde é atribuído à aprendizagem dos indivíduos um sentido de "pertença"; e, por fim, identidade, resultante de um processo de construção da história individual e das aprendizagens pessoais que o indivíduo obtém dentro da própria comunidade.

As interações e as experiências dos treinadores dentro de uma comunidade de prática desencadeiam em seus participantes o sentimento de "pertencer", o que de fato influencia no engajamento e na disponibilidade para a aprendizagem (LEMYRE; TRUDEL; DURAND-BUSH, 2007). Ademais, o compartilhamento de informações através dessa rede de relacionamentos possibilita a troca de experiências e conhecimentos entre os profissionais, principalmente em eventos sociais vinculados à modalidade esportiva e na relação com outros treinadores durante as competições (WRIGHT; TRUDEL; CULVER, 2007).

\section{IMPLICAÇÕES NO PROCESSO DE APRENDIZAGEM PROFISSIONAL DOS TREINADORES}

Ao longo de sua trajetória, a aprendizagem do treinador decorre da combinação de um número variado de situações e contextos em diferentes períodos de sua vida pessoal e profissional (CUSHION; NELSON, 2013).

A experiência como atleta e o mentoring são situações de aprendizagem mais comuns num período que vai desde suas primeiras experiências como esportista até assumir o papel de treinador. Nessas situações não há uma procura intencional do conhecimento, portanto, o treinador só toma consciência dos conhecimentos adquiridos em momento futuro. Já na aprendizagem através do compartilhamento de informações com outros treinadores e da experiência de prática profissional é o treinador quem busca as informações úteis as suas necessidades laborais, ou seja, trata-se de iniciativas mais conscientes que as anteriores (CUNHA; ESTRIGA; BATISTA, 2014). 
A aprendizagem do treinador parece não estar necessariamente ligada a determinada estrutura de conhecimentos e estratégias de ensino previamente definidas do que se deve aprender para se tornar um treinador. Trata-se de um percurso de natureza híbrida, marcado por diversas interações sociais, que ora tendem a ocorrer em um contexto mais formal e ora num contexto mais informal, onde prevalecem os interesses do próprio indivíduo. Isto significa que os treinadores têm vislumbrado seus próprios caminhos para se tornarem e consolidarem enquanto treinadores, resultando em um tipo de "currículo individual" ou "currículo personalizado" de aprendizagem em longo prazo (MIELKE, 2007).

Os conhecimentos, as crenças, as concepções e os comportamentos do treinador são adquiridos no "mundo real", por meio do que viu, do que fez, do que sentiu e do que pensou, desde o período em que teve os primeiros contatos com a prática esportiva estendendo-se no decorrer de sua vida, ou seja, as experiências vividas pelo próprio indivíduo é que fundamentam essa aprendizagem pessoal num contexto social.

Em particular, as experiências esportivas têm implicações importantes na atuação do treinador, pois parece que possibilitam criar crenças ou um repertório de "modos de ação" sobre como ensinar a partir das percepções de suas próprias aprendizagens, das observações que realiza de outros treinadores a ensinar e da resolução de situações enfrentadas no seu cotidiano de intervenção prática.

A reflexão sobre esses episódios, as dificuldades e situações de êxito na aprendizagem pessoal e no ensino podem aumentar a capacidade do treinador de lidar com as próprias dificuldades encontradas da prática profissional. Ele pode utilizar os eventos passados, guardados na sua memória, como um precedente ou exemplo para sua intervenção, auxiliando-o a perceber uma situação como similar a outra, mas não idêntica, ou seja, esse confronto constante contribui no sentido de gerar um senso de continuidade entre as práticas ao longo do tempo. Para Gilbert, Côté e Mallet (2006), a aprendizagem por meio da experiência em contextos informais é de vital importância pela quantidade relativamente pequena de tempo que um treinador pode gastar em um determinado ambiente de aprendizagem formal, comparada ao número de horas que ele passa no ambiente esportivo treinando e interagindo com os atletas e outros treinadores.

As experiências no contexto social esportivo informal envolvem a partilha de conhecimentos e crenças, bem como a reflexão e a aprovação dos conhecimentos construídos (GALIPEAU; TRUDEL, 2006). Além disso, o envolvimento na comunidade esportiva proporciona, simultaneamente, a reconstrução constante de significados e identidades do treinador através das ações cotidianas das relações sociais, das rotinas, das conversas, do fazer junto, dos gestos, das estórias e das ações decorrentes da participação ativa nessa comunidade (WENGER, 1998).

Ainda no papel de jovem esportista, ele aprende ao observar a intervenção de seu treinador, posteriormente, no papel de auxiliar ou assistente, aprende observando e dialogando com o treinador principal mais experiente a atuar, e logo passa a observar outros treinadores adversários ou pares, já na função de treinador principal.

Apesar de o acúmulo dessas experiências na memória do treinador fornecer determinada segurança para desenvolver seu papel, sobretudo no início de carreira, ele não garante a qualidade da intervenção. A sua competência profissional está relacionada à capacidade de 
perceber e interpretar os problemas situacionais de prática, assim como de selecionar as estratégias mais adequadas para solucioná-los (GILBERT; TRUDEL, 2001). A aprendizagem, neste caso, implica um processo social de aceitação e assimilação de conhecimentos, valores, crenças e expectativas da cultura esportiva e, por outro lado, um processo mais individual que implica uma aprendizagem "profunda", na qual o treinador ressignifica suas concepções sobre seu papel e seus comportamentos, influenciando de forma significativa a sua prática.

A importância de se aprender "na" experiência e, principalmente, "com" a experiência, de acordo com Anderson, Knowles e Gilbourne (2004), decorre de um processo de reflexão no qual o treinador deve examinar e interpretar suas próprias experiências, transformando-as em conhecimentos úteis para sua prática. Trata-se do envolvimento ativo num processo de "garimpar" o conhecimento incorporado no interior da própria experiência. Assim, todas as ações, sensações, emoções e imagens experimentadas e memorizadas pelos treinadores desde a infância vão, ao longo do tempo, balizando a incorporação de crenças e comportamentos sobre os significados e ações do que é ser treinador. Em outros termos, é um tipo de "personalização" das experiências, ou seja, aquilo que o indivíduo experimenta e observa é memorizado e logo integrado a sua própria biografia (JARVIS, 2006).

Portanto, as experiências acumuladas devem preparar o indivíduo para experiências posteriores, de qualidade mais profunda e mais ampla do que a anterior, configurando-se em um processo contínuo de crescimento e aprendizagem a partir da reconstrução da própria intervenção. Neste processo, torna-se central a interação de mecanismos internos do indivíduo e dos atributos externos de ordem sociocultural, porém, diante da dificuldade de isolar aspectos particulares do indivíduo da participação da pessoa no ambiente, a interação de diversas formas com outras pessoas parece representar os atributos deste processo (JARVIS, 2006).

Contudo, o entendimento a respeito do que implica determinadas situações e contextos de aprendizagem na formação profissional do treinador possibilita discutir a aprendizagem, tanto na perspectiva do próprio treinador-aluno (situação de aprendizagem) como na dos locais ou ambientes de aprendizagem (instituições, organizações, cursos, trabalho) (TRUDEL; CULVER; WERTHNER, 2013).

Acredita-se que se a busca por projeções futuras no âmbito da formação profissional do treinador tem como referência a natureza das situações (mediada, não mediada, interna) e dos contextos de aprendizagem (formal, não formal, informal), deve-se considerar como elementos fundamentais: a pessoa do treinador (como aluno); a situação social em que ocorre a aprendizagem; a experiência que ele tem de tal situação; e o processo de transformá-la e incorporá-la à biografia do treinador (JARVIS, 2006).

\section{CONSIDERAÇÕES FINAIS}

A aprendizagem profissional do treinador pode ser entendida como um processo de socialização esportiva que inicia na infância e se estende ao longo da vida. Apesar de não haver uma única trajetória de aprendizagem para os treinadores, destacam-se algumas situações marcantes durante esse percurso, nomeadamente a entrada no contexto esportivo; a permanência e o grau de significados pessoais atribuídos às experiências esportivas; 0 ingresso ou transição da condição de praticante para a de treinador; e a obtenção de conhecimento para se manter no papel de treinador. Embora essa trajetória tenha marcadamente um caráter 
individual ou pessoal, há também na atualidade o reconhecimento do importante condicionante contextual ou da cultura esportiva no direcionamento da carreira do treinador.

Apesar da participação em diferentes iniciativas de formação implementadas nos contextos Formal e Não formal, investigações apontam que o treinador tem buscado a proficiência para sua intervenção pedagógica, de modo preponderante, em situações de aprendizagem que são tipicamente de um contexto Informal. Nessas situações, o engajamento nas fases mais jovens refere-se à aprendizagem de prática enquanto jogador, e o engajamento no papel de treinador refere-se, primordialmente, à aprendizagem para ensinar.

Independentemente da fase da vida do treinador, esse engajamento pessoal contribui para a construção de um repertório de "modelos de intervenção", isto é, exemplos de ações para resolver suas tarefas pedagógicas enquanto treinador. Ao se deparar com as demandas de seu ambiente de intervenção, o treinador mobiliza em sua memória episódica tais "modelos" (experiências prévias) e, ao atribuir-Ihes novos significados, elabora um tipo de "cópia personalizada" dessas experiências, úteis para realizar suas tarefas dentro de um contexto específico de prática profissional.

Assim, acredita-se que a criação de situações de aprendizagem informal em contextos institucionalizados ou programas de formação de treinadores, oferecidos tanto por associações e federações esportivas ou em cursos de formação inicial universitária em Educação Física, contribui para ampliar as possibilidades de aprendizagem profissional dos treinadores.

\section{REFERÊNCIAS}

ANDERSON, Ailsa; KNOWLES, Zoe; GILBOURNE, David. Reflective practice for applied sport psychologists: a review of concepts, models, practical implications and thoughts on dissemination. The Sport Psychologist, Champaign, v. 18, n. 2, p. 188-203, 2004.

BAILEY, Richard; COPE, Edward; PEARCE, Gemma. Why do children take part in, and remain involved in sport? A literature review and discussion of implications for sports coaches. International Journal of Coaching Science, Leeds, v. 7, n. 1, p. 56-75, 2013.

BANDURA, Albert et al. (Org.). Teoria social cognitiva: conceitos básicos. Porto Alegre: Artmed, 2008.

CALLARY, Bettina; WERTHNER, Penny; TRUDEL, Pierre. How meaningful episodic experiences influence the process of becoming an experienced coach. Qualitative Research in Sport, Exercise and Health, Champaign, v. 4, n. 3, p. 420-438, Nov. 2012.

CASSIDY, Tania; JONES, Robyn; POTRAC, Paul. Understanding sports coaching: the social, cultural and pedagogical foundations of coaching practice. London: Routledge Abingdon, 2004.

CÔTÉ, Jean; HAY, John. Family influences on youth sport participation and performance. In: SILVA, J. M.; STEVENS, D. Psychological foundations of sport. Boston: Merrill, 2002. p. 503-519.

CROPLEY, Brendan; HANTON, Sheldon. The role of the reflective practice in applied sport psychology: contemporary issues for professional practice. In: HANTON, Sheldon; MELLALIEU, 
Stephen. Professional practice in sport psychology: a review. London: Routledge, 2011. p. 307-336.

CUNHA, Ana Felipa V. P.; ESTRIGA, Maria Luísa Dias; BATISTA, Paula Maria Fazendeiro. Fontes de conhecimento percebidas pelos treinadores: estudo com treinadores de andebol da $1^{\text {a }}$ divisão seniores masculinos em Portugal. Movimento, Porto Alegre, v. 20, n. 3, p. 917-940, jul./ set. 2014.

CULVER, Diane; TRUDEL, Pierre. 'Cultivating coaches' communities of practice. In: JONES, Robyn. The sports coach as educator: re-conceptualizing sports coaching. London: Routledge, 2006. p. 97-112.

CUSHION, Christopher et al. Coach learning and development: a review of literature. London: Leeds Sports Coach UK, 2010.

CUSHION, Christopher. Mentoring: Harnessing the power of expertise. In: JONES, Robyn. The sports coach as educator: re-conceptualizing sports coaching. London: Routledge, 2006. p. 128-144.

CUSHION, Christopher; ARMOUR, Kathy; JONES, Robyn. Coach education and continuing professional development: experience and learning to coach. Quest, Illinois, n. 46, p. 153-163, 2003.

CUSHION, Christopher; NELSON, Lee. Coach education and learning: developing the field. In: POTRAC, Paul; GILBERT, Wade; DENISON, Jim. Routledge handbook of sports coaching. London: Routledge, 2013.p. 359-374.

FRASER-THOMAS, Jessica; CÔTÉ, Jean. Understanding adolescents' positive and negative developmental experiences in sport. The Sport Psychologist, Champaign, v. 23, n. 1, p. 3-23, 2009.

GALIPEAU, James; TRUDEL, Pierre. Athlete learning in a community of practice: is there a role for the coach? In: JONES, Robyn. The sports coach as educator: re-conceptualizing sports coaching. London: Routledge, 2006. p. 77-94.

GILBERT, Wade; CÔTÉ, Jean; MALLET, Clifford. Developmental paths and activities of successful sport coaches. International Journal of Sport Science and Coaching, Leeds, v. 1, n. 1, p. 69-76, 2006.

GILBERT, Wade; TRUDEL, Pierre. Learning to coach through experience: reflection in model youth sport coaches. Journal of Teaching in Physical Education, Champaign, v. 21, n. 1, p. 16-34, 2001.

IRWIN, Gareth; HANTON, Sheldon; KERWIN, David. Reflective practice and the origins of elite coaching knowledge. Reflective Practice, London, v. 5, n. 3, p. 425-442, Oct. 2004.

JARVIS, Peter. Towards a comprehensive theory of human learning. London: Routledge, 2006.

JONES, Robyn; ARMOUR, Kathy; POTRAC, Paul. Sports coaching cultures: from practice to theory. London: Routledge, 2004.

KEEGAN, Richard et al. A qualitative investigation exploring the motivational climate in earlycareer sports participants: coach, parent and peer influences on sport motivation. Psychology of Sport and Exercise, London, v. 10, n. 3, p.361-372, 2009. 
LAVE, Jean; WENGER, Etienne. Situated learning: legitimate peripheral participation. Cambridge: Cambridge University Press, 1991.

LEMYRE, François; TRUDEL, Pierre; DURAND-BUSH, Natalie. How youth-sport coaches learn to coach. The Sport Psychologist, Champaign, v. 21, n. 2, p. 191-209, 2007.

MIELKE, Danny. Coaching experience, playing experience and coaching tenure. International Journal of Sports Science and Coaching, Leeds, v. 2, n. 2, p. 105-108, 2007.

MONTERO, Lourdes. A construção do conhecimento profissional docente. Lisboa: Instituto Piaget, 2005.

MOON, Jennifer A. A handbook of reflection and experiential learning: theory and practice. London: Kogan Page, 2004.

NASH, Christine; COLLINS, Dave. Tacit Knowledge in Expert Coaching: Science or Art? Quest, Illinois, v. 58, n. 4, p. 465-477, 2006.

NELSON, Lee; CUSHION, Christopher; POTRAC, Paul. Formal, non formal and informal coach learning: a holistic conceptualization. International Journal of Sports Science and Coaching, Leeds, v. 1, n. 3, p. 247-259, 2006.

RAMOS, Valmor et al. A aprendizagem profissional: as representações de treinadores desportivos de jovens: quatro estudos de caso. Motriz, Rio Claro, v. 17, n. 2, p. 280-291, 2011.

RAMOS, Valmor; BRASIL, Vinicius Zeilmann; GODA, Ciro. A aprendizagem profissional na percepção de treinadores de jovens surfistas. Revista da Educação Física/UEM, Maringá, v. 23, n. 3, p. 431-442, 2012.

SALMELA, John. Learning from the development of expert coaches. Coaching and Sport Science Journal, Illinois, v. 1, n. 3, p. 1-11, 1994.

SCHINKE, Robert; BLOOM, Gordon; SALMELA, John. The career stages of elite Canadian basketball coaches. AVANTE, Champaign, v. 1, n. 1, p. 48-62, 1995.

SCHÖN, Donald. Educando o profissional reflexivo: um novo design para o ensino e a aprendizagem. Porto Alegre: Artmed, 2000.

SCHWARTZ, Shalom. Valores Humanos Básicos: seu contexto e estrutura intercultural. In: TAMAYO Álvaro, PORTO Juliana Barreiros (Org.). Valores e comportamento nas organizações. Petrópolis: Vozes, 2005. p. 21-55.

SFARD, Anna. On two metaphors for learning and the dangers of choosing just one. Educational Researcher, Champaign, v. 27, n. 2, p. 4-13, 1998.

SOLER, Maria Rosa. Mentoring: Estrategia de desarrollo de recursos humanos. Barcelona: Gestion 2000, 2003.

TALAMONI, Guilherme Augusto; OLIVEIRA, Flávio Ismael da Silva; HUNGER, Dagmar. As configurações do futebol brasileiro: análise da trajetória de um treinador. Movimento, Porto Alegre, v. 19, n. 1, p. 73-93, 2013.

TRUDEL, Pierre; CULVER, Diane; WERTHNER, Penny. Looking at coach development from the coach-learner's perspective: considerations for coach development administrators. In: POTRAC, Paul; GILBERT, Wade; DENISON, Jim. Routledge handbook of sports coaching. London: 
Routledge, 2013. p. 375-387.

TRUDEL, Pierre; GILBERT, Wade. Coaching and coach education. In: KIRK, David; O'SULLIVAN, Mary; MCDONALD, Doune (Ed.). Handbook of physical education. London: Sage, 2006. p. 516-539.

WEAVER, Margie; CHELLADURAI, Packianathan. A mentoring model for management in sport and physical education. Quest, Illinois, v. 51, n. 1, p. 24-38, 1999.

WENGER, Etienne. Communities of practice: learning, meaning and identity. Cambridge: Cambridge University Press, 1998.

WERTHNER, Penny; TRUDEL, Pierre. A new theoretical perspective for understanding how coaches learn to coach. The Sport Psychologist, Champaign, v. 20, n. 2, p. 198-212, 2006.

WRIGHT, Trevor; TRUDEL, Pierre; CULVER, Diane. Learning how to coach: the different learning situations reported by youth ice hockey coaches. Physical Education and Sport Pedagogy, Worcester, v. 12, n. 3, p. 127-144, 2007. 